\title{
VPPP Algorithms with Multiple Antennas by Applying MADOCA Products
}

\author{
Hirokazu Hasegawa, Dai Mimura, Yoshiki Shibayama, Yukihiro Kubo and Sueo Sugimoto \\ Department of Electrical and Electronic Engineering, Ritsumeikan University \\ 1-1-1 Noji-Higashi, Kusatsu City, Shiga 525-8577 Japan \\ Tel: +81-(0)77-561-2673, FAX: +81-(0)77-561-2663 \\ E-mail: sugimoto@se.ritsumei.ac.jp
}

\begin{abstract}
According to our previous research works, we present the algorithms of the VPPP (Very Precise Point Positions) using multiple GNSS antennas with known distances each other. VPPP algorithms contain the very precise GNSS positioning as well as the so-called GNSS Gyro by estimating the multiple baseline vectors from the reference antenna to other antennas. In this paper, we attempt to implement the satellite orbits and clocks correction data from the MADOCA Products which had been developed by JAXA, into our VPPP algorithms. Further, finally, we show the positioning accuracy as well as the estimation accuracy of the Euler's angles in the GNSS Gyro, in comparison with using the so-called GPS broadcast ephemeris.
\end{abstract}

\section{Introduction}

In this paper, according to our previous research works [1][4], we present the algorithms of the VPPP (Very Precise Point Positions) using multiple GNSS antennas with known distances each other. VPPP algorithms contain the very precise GNSS positioning as well as the so-called GNSS Gyro by estimating the multiple baseline vectors from the reference antenna to other antennas. In this paper, we implement the satellite orbits and clocks correction data from the MADOCA (Multi-GNSS Advanced Demonstration tool for Orbit and Clock Analysis) Products which had been developed by JAXA (Japan Aerospace Exploration Agency) and are provided by GPAS (Global Positioning Augumentation Service Corporation) [6]-[8], into our VPPP algorithms. Further, finally, we show the positioning accuracy as well as the estimation accuracy of the Euler's angles in the GNSS Gyro, in comparison with using the so-called GPS broadcast ephemeris.

First of all, in this paper, we review the previous VPPP algorithms with multiple antennas [1]-[4] based on Global navigation satellite system Regression models (referred as GR models) which have been developed in [5].

By presenting GR equations according to [5], in this paper, we derive very precise point positioning (VPPP) algorithms with using multiple antennas which are disposed with solid geometrical distances.
In the next section, we review the GR models and use them to derive VPPP algorithms in [2]. Further, in the section 3, we review the VPPP algorithms [3]. based on the double difference (DD) observables by each pair of two antennas and apply the algorithms for estimating baseline vectors and GNSS Gyro [4].

\section{GNSS Regression Models with Contraints}

Similarly to [5], we only describe the case of L1 frequncy GNSS Regresion (GR) models for the observed positioning data consisting of L1 carrier phases and pseudoranges based on C/A code due to simpler description and the commercial usage of positioning. The natural extensions of GNSS regression models for multiple frequencies of Galileo, Compass/BeiDou, and GLONASS are also similarly formulated. Namely, we consider the following fundamental measurements of L1 band carrier phases $\varphi_{L 1, u}^{p}(t)$; and the pseudoranges $\rho_{C A, u}^{p}(t)$ based on C/A codes, respectively, as follows:

$$
\begin{aligned}
\rho_{C A, u}^{p}(t)= & r_{u}^{p}\left(t, t-\tau_{u}^{p}\right)+\delta I_{u}^{p}(t)+\delta T_{u}^{p}(t) \\
& +c\left[\delta t_{u}(t)-\delta t^{p}\left(t-\tau_{u}^{p}\right)\right] \\
& +\delta b_{C A, u}-\delta b_{C A}^{p}+e_{C A, u}^{p}(t), \\
\Phi_{L 1, u}^{p}(t)= & \lambda_{1} \varphi_{L 1, u}^{p}(t) \\
= & r_{u}^{p}\left(t, t-\tau_{u}^{p}\right)-\delta I_{u}^{p}(t)+\delta T_{u}^{p}(t) \\
& +c\left[\delta t_{u}(t)-\delta t^{p}\left(t-\tau_{u}^{p}\right)\right] \\
& +\delta b_{L 1, u}-\delta b_{L 1}^{p}+\lambda_{1} N_{u}^{p}+\lambda_{1} \varepsilon_{u}^{p}(t) .
\end{aligned}
$$

where $c\left(\approx 2.99792458 \times 10^{8}[\mathrm{~m}]\right)$ denotes the speed of light, and $f_{1}$ and $\lambda_{1}$ are the central frequency and the wave length of the L1 carrier wave

$$
f_{1}=2 \times 77 \times 10.23[\mathrm{MHz}]=1575.42[\mathrm{MHz}]
$$

In (1)-(2), the so-called receiver's biases, $\left\{\delta b_{C A, u}, \delta b_{L 1, u}\right\}$, and the satellite biases, $\left\{\delta b_{C A}^{p}, \delta b_{L 1}^{p}\right\}$, are contained in the usual observed positioning data consisting of L1 carrier phase and the pseudorange based on C/A codes. Also $r_{u}^{p}\left(t, t-\tau_{u}^{p}\right)$ is the geometric distance between the receiver $u$ at the time $t$ and the satellite $p$ at the time $t-\tau_{u}^{p}\left(\tau_{u}^{p}\right.$ denotes the travel time from the satellite $p\left(p=1, \ldots, n_{s}\right)$ to 
the receiver $u$ ). Namely,

$$
\begin{aligned}
r_{u}^{p}(t) \equiv & r_{u}^{p}\left(t, t-\tau_{u}^{p}\right) \\
= & {\left[\left(x_{u}(t)-x^{p}\left(t-\tau_{u}^{p}\right)\right)^{2}+\left(y_{u}(t)-y^{p}\left(t-\tau_{u}^{p}\right)\right)^{2}\right.} \\
& \left.+\left(z_{u}(t)-z^{p}\left(t-\tau_{u}^{p}\right)\right)^{2}\right]^{1 / 2} \\
= & \left\|u(t)-s^{p}\left(t-\tau_{u}^{p}\right)\right\|
\end{aligned}
$$

where $u \equiv\left[x_{u}, y_{u}, z_{u}\right]^{\mathrm{T}}$ and $s^{p} \equiv\left[x^{p}, y^{p}, z^{p}\right]^{\mathrm{T}}$ are a user (unknown) and satellite positions, respectively. Also $n_{s}$ shows the number of the observable satellites. Further in (1), (2), $\delta I_{u}^{p}(t)$ and $\delta T_{u}^{p}(t)$ reflect the delay or the advance associated with the transmission of the L1 signal through the ionosphere and the troposphere, respectively. $\delta t_{u}(t)$ and $\delta t^{p}\left(t-\tau_{u}^{p}\right)$ are the clock errors of the receiver $u$ at the time $t$ and the satellite $p$ at the time $t-\tau_{u}^{p} . N_{u}^{p}$ denotes integer ambiguity between the satellite $p$ and the receiver $u$, and $\varepsilon^{p}(t), e^{p}(t)$ denote measurement errors.

Eq. (4) contains the satellite orbital errors. The estimated satellite orbits are obtained from the navigation messages which are decoded from the transmitted L1 signal, and in this paper, especially we use the MADOCA Product which are developed by JAXA and are transmitted from QuasiZenith Satellite System (QZSS) [6]-[8] Let us denote $\hat{s}^{p}$ as the estimated position of the satellite $s^{p}$ at the time $t-\tau_{u}^{p}$.

We use the following relations of the derivatives

$$
\begin{array}{ll}
\frac{\partial r_{u}^{p}}{\partial x_{u}}=\frac{\left(x_{u}-x^{p}\right)}{r_{u}^{p}}, & \frac{\partial r_{u}^{p}}{\partial y_{u}}=\frac{\left(y_{u}-y^{p}\right)}{r_{u}^{p}}, \\
\frac{\partial r_{u}^{p}}{\partial z_{u}}=\frac{\left(z_{u}-z^{p}\right)}{r_{u}^{p}}, & \left(p=1,2, \ldots, n_{s}\right),
\end{array}
$$

and also

$$
\begin{array}{ll}
\frac{\partial r_{u}^{p}}{\partial x^{p}}=-\frac{\left(x_{u}-x^{p}\right)}{r_{u}^{p}}, & \frac{\partial r_{u}^{p}}{\partial y^{p}}=-\frac{\left(y_{u}-y^{p}\right)}{r_{u}^{p}}, \\
\frac{\partial r_{u}^{p}}{\partial z^{p}}=-\frac{\left(z_{u}-z^{p}\right)}{r_{u}^{p}}, & \left(p=1,2, \ldots, n_{s}\right) .
\end{array}
$$

Then we have the relation:

$$
\frac{\partial r_{u}^{p}}{\partial u}=-\frac{\partial r_{u}^{p}}{\partial s^{p}}
$$

Thus the 1st order Taylor series approximation of (4) around the previous estimated value $u=\hat{u}^{(\nu)}$ and $s^{p}=\hat{s}^{p}$ is given by

$$
\begin{aligned}
r_{u}^{p} & \cong r_{\hat{u}^{(\nu)}}^{\hat{p}}+g_{\hat{u}^{(\nu)}}^{\hat{p}}\left[u-s^{p}-\left(\hat{u}^{(\nu)}-\hat{s}^{p}\right)\right] \\
& =\left\|\hat{u}^{(\nu)}-\hat{s}^{p}\right\|+\frac{\left(\hat{u}^{(\nu)}-\hat{s}^{p}\right)^{\mathrm{T}}}{\left\|\hat{u}^{(\nu)}-\hat{s}^{p}\right\|}\left[u-s^{p}-\left(\hat{u}^{(\nu)}-\hat{s}^{p}\right)\right] \\
& =\frac{\left(\hat{u}^{(\nu)}-\hat{s}^{p}\right)^{\mathrm{T}}}{\left\|\hat{u}^{(\nu)}-\hat{s}^{p}\right\|}\left(u-s^{p}\right)
\end{aligned}
$$

for $p=1,2, \ldots, n_{s}$, where

$$
g_{\hat{u}^{(\nu)}}^{\hat{p}} \equiv\left[\frac{\partial r_{u}^{p}}{\partial u}\right]_{u=\hat{u}^{(\nu)}, s^{p}=\hat{s}^{p}}^{\mathrm{T}}=\frac{\left(\hat{u}^{(\nu)}-\hat{s}^{p}\right)^{\mathrm{T}}}{\left\|\hat{u}^{(\nu)}-\hat{s}^{p}\right\|}
$$

From (1)-(2), therefore, we have the approximations:

$$
\begin{aligned}
\rho_{C A, u}^{p} \cong & g_{\hat{u}^{(\nu)}}^{\hat{p}}\left(u-s^{p}\right) \\
& +\delta I_{u}^{p}+\delta T_{u}^{p}+c\left(\delta t_{u}-\delta t^{p}\right) \\
& +\delta b_{C A, u}-\delta b_{C A}^{p}+e_{C A, u}^{p}, \\
\Phi_{L 1, u}^{p} \cong & g_{\hat{u}^{(\nu)}}^{\hat{p}}\left(u-s^{p}\right) \\
& -\delta I_{u}^{p}+\delta T_{u}^{p}+c\left(\delta t_{u}-\delta t^{p}\right) \\
& +\delta b_{L 1, u}-\delta b_{L 1}^{p}+\lambda_{1} N_{L 1, u}^{p}+\lambda_{1} \varepsilon_{L 1, u}^{p},
\end{aligned}
$$

Define the $n_{s} \times 3$ matrix:

$$
G_{\hat{u}(\nu)}^{\hat{s}} \equiv\left[\begin{array}{c}
g_{\hat{\hat{u}}(\nu)}^{\hat{1}} \\
g_{\hat{u}(\nu)}^{2} \\
\vdots \\
g_{\hat{u}^{(\nu)}}^{\hat{n}_{s}}
\end{array}\right],
$$

namely,

$$
G_{\hat{u}^{(\nu)}}^{\hat{s}}=\left[\begin{array}{ccc}
\frac{\partial r_{\hat{u}^{(\nu)}}^{\hat{1}}}{\partial \hat{x}_{u}^{(\nu)}} & \frac{\partial r_{\hat{u}^{(\nu)}}^{\hat{1}}}{\partial \hat{y}_{u}^{(\nu)}} & \frac{\partial r_{\hat{u}^{(\nu)}}^{\hat{u}^{(\nu)}}}{\partial \hat{z}_{u}^{(\nu)}} \\
\frac{\partial r_{\hat{u}^{(\nu)}}^{\hat{2}}}{\partial \hat{x}_{u}^{(\nu)}} & \frac{\partial r_{\hat{u}^{(\nu)}}^{\hat{2}}}{\partial \hat{y}_{u}^{(\nu)}} & \frac{\partial r_{\hat{u}^{(\nu)}}^{\hat{2}}}{\partial \hat{z}_{u}^{(\nu)}} \\
\vdots & \vdots & \vdots \\
\frac{\partial r_{\hat{u}_{s}^{(\nu)}}^{\hat{n}_{s}}}{\partial \hat{x}_{u}^{(\nu)}} & \frac{\partial r_{\hat{u}^{(\nu)}}^{\hat{n}_{s}}}{\partial \hat{y}_{u}^{(\nu)}} & \frac{\partial r_{\hat{u}_{s}^{(\nu)}}^{\hat{n}_{s}}}{\partial \hat{z}_{u}^{(\nu)}}
\end{array}\right] .
$$

The satellite's hardware biases $\delta b^{p}$ are compensated and receiver's hardware biases $\delta b_{*, u}$ are estimated by defining the terms the $2 \times 1$ vector

$$
\delta b_{u} \equiv\left[\delta b_{C A, u}, \delta b_{L 1, u}\right]^{\mathrm{T}} .
$$

Furthermore, from (12), we define a block diagonal matrix with the size $\left(n_{s} \times 3 n_{s}\right)$ :

$$
G_{D, \hat{u}^{(\nu)}}^{\hat{s}} \equiv\left[\begin{array}{ccccc}
g_{\hat{u}^{(\nu)}}^{\hat{1}} & O & O & \cdots & O \\
O & g_{\hat{u}^{(\nu)}}^{\hat{2}} & O & \cdots & O \\
\vdots & & \ddots & & \vdots \\
\vdots & & & \ddots & O \\
O & \cdots & \cdots & O & g_{\hat{u}^{(\nu)}}^{\hat{n}_{s}}
\end{array}\right] .
$$

Then from (10)-(15), we have the following vector matrix regression equation:

$$
y_{\hat{u}^{(\nu)}}=H_{\hat{u}^{(\nu)}} \theta_{u}+v_{u},
$$

where

$$
y_{\hat{u}^{(\nu)}} \equiv\left[\begin{array}{c}
\rho_{C A, \hat{u}^{(\nu)}} \\
\Phi_{L 1, \hat{u}^{(\nu)}}
\end{array}\right], \quad \theta_{u} \equiv\left[\begin{array}{c}
u \\
c \delta t_{u} \\
\delta b_{u} \\
s \\
c \delta t^{s} \\
\delta I_{u} \\
\delta T_{u} \\
\lambda_{1} N_{L 1, u}
\end{array}\right]
$$


Then we get some knowledge of the satellite position $s$, the satellite clock error $c \delta t^{s}$ as well as the delay or the advance due to the ionospheric and tropospheric effects, $\delta I_{u}^{\mathrm{T}}$ and $\delta T_{u}^{\mathrm{T}}$ are, for instance,

$$
\begin{aligned}
\tilde{s} & =s+e_{s}, \\
c \tilde{\delta} t^{s} & =c \delta t^{s}+e_{\delta t^{s}}, \\
\tilde{\delta I_{u}} & =\delta I_{u}+e_{\delta I_{u}}, \\
\tilde{\delta T_{u}} & =\delta T_{u}+e_{\delta T_{u}} .
\end{aligned}
$$

Substituting the above relations into the GR equation (16), and neglect $s, c \delta t^{s}, \delta I_{u}, \delta T_{u}$, we have

$$
\left[\begin{array}{c}
y_{C A, \hat{u}^{(\nu)}}^{\hat{s}} \\
y_{L 1, \hat{u}^{(\nu)}}^{\hat{s}}
\end{array}\right]=C_{\hat{u}^{(\nu)}}^{\hat{s}}\left[\begin{array}{c}
u \\
c \delta t_{u} \\
\delta b_{u} \\
\lambda_{1} N_{L 1, u}
\end{array}\right]+v
$$

where

$$
\begin{aligned}
& C_{\hat{u}^{(\nu)}}^{\hat{s}}=\left[\begin{array}{lllll}
G_{\hat{u}^{(\nu)}}^{\hat{s}} & \mathbf{1} & \mathbf{1} & & O \\
G_{\hat{u}^{(\nu)}}^{\hat{s}} & \mathbf{1} & & \mathbf{1} & I
\end{array}\right], \\
& v=\left[\begin{array}{c}
G_{D, \hat{u}^{(\nu)}}^{\hat{s}} e_{s}+e_{\delta t^{s}}-e_{\delta I_{u}}-e_{\delta T_{u}}+e_{C A, u} \\
G_{D, \hat{u}^{(\nu)}}^{\hat{s}} e_{s}+e_{\delta t^{s}}+e_{\delta I_{u}}-e_{\delta T_{u}}+\lambda_{1} \varepsilon_{L 1, u}
\end{array}\right],
\end{aligned}
$$

and

$$
\begin{aligned}
y_{C A, \hat{u}^{(\nu)}}^{\hat{s}} & =\rho_{C A, u}^{s}+G_{D, \hat{u}^{(\nu)}}^{\hat{s}}+c \tilde{\delta} t^{s}-\tilde{\delta I} \tilde{I}_{u}-\tilde{\delta T}{ }_{u} \\
y_{L 1, \hat{u}^{(\nu)}}^{\hat{s}} & =\Phi_{L 1, u}^{s}+G_{D, \hat{u}^{(\nu)}}^{\hat{s}}+c \tilde{\delta} t^{s}+\tilde{\delta I_{u}}-\tilde{\delta T_{u}}
\end{aligned}
$$

\subsection{Very Precise Point Positioning}

Now let us consider that multiple antennas $u_{i}, i=1, \ldots, n_{r}$ are disposed with the given distance $d_{j i}$ between any two antennas $u_{i}$ and $u_{j}$. In this assumption, we have the following $n_{r}$ single frequency GR equations:

$$
y_{\hat{u}_{i}^{(\nu)}}=C_{\hat{u}_{i}^{(\nu)}}^{\hat{s}_{i}} \theta_{u_{i}}+v_{u_{i}},
$$

for $i=1, \cdots, n_{r}$, where

$$
\theta_{u_{i}}=\left[\begin{array}{c}
u_{i} \\
c \delta t_{u_{i}} \\
\delta b_{u_{i}} \\
\lambda_{1} N_{L 1, u_{i}}
\end{array}\right]
$$

with the constraint

$$
\begin{aligned}
& d_{j i}=\left\|u_{j}-u_{i}\right\|+\text { small noise } \\
&=\sqrt{\left(x_{i}-x_{j}\right)^{2}+\left(y_{i}-y_{j}\right)^{2}+\left(z_{i}-z_{j}\right)^{2}} \\
&+ \text { small noise. }
\end{aligned}
$$

Now we show state equations and measurement equations for applying Klman filtering. Let us show the Kalman filtering algorithm for VPPP with the constraint conditions in (27). For this purpose, we show the state equations for the static case. Let us show the state equation for each component in (26).

\subsubsection{State equation for receiver's clock error}

Also the receiver's clock errors are generally modeled as follows $[11,12,13]$ (let us call the following model as the Amodel of the receiver's clock error),

$$
\begin{aligned}
c \delta t_{u, t+1} & =c \delta t_{u, t}+\Delta_{t} c \dot{\delta} t_{u, t}+w_{c \delta t_{u}, t} \\
c \dot{\delta} t_{u, t+1} & =c \dot{\delta} t_{u, t}+w_{c \dot{\delta} t_{u}, t},
\end{aligned}
$$

where $\Delta_{t}$ denotes the sampling interval of the receiver's clock error, and the noise $w_{c \delta t_{u}, t}$ and $w_{c \dot{\delta} t_{u}, t}$ are assumed as white Gaussian processes with zero means and covariances $q_{c \delta t}$ and $q_{c \dot{\delta} t}$, respectively. Then we can write

$$
\begin{aligned}
{\left[\begin{array}{c}
c \delta t_{u, t+1} \\
\dot{\delta} t_{u, t+1}
\end{array}\right] } & =\left[\begin{array}{cc}
1 & \Delta_{t} \\
0 & 1
\end{array}\right]\left[\begin{array}{c}
c \delta t_{u, t} \\
c \dot{\delta} t_{u, t}
\end{array}\right]+\left[\begin{array}{l}
w_{c \delta t_{u}, t} \\
w_{c \dot{\delta} t_{u}, t}
\end{array}\right] \\
& \equiv F_{A, c \delta t}\left[\begin{array}{c}
c \delta t_{u, t} \\
c \dot{\delta} t_{u, t}
\end{array}\right]+\left[\begin{array}{c}
w_{c \delta t_{u}, t} \\
w_{c \dot{\delta}_{u}, t}
\end{array}\right]
\end{aligned}
$$

Also we consider another statistical model of the receiver's clock error from the standpoint of time-series analysis. Namely we assume the time derivative of the receiver's clock error is a first order markov process as follows:

$$
\begin{aligned}
c \delta t_{u, t+1} & =c \delta t_{u, t}+\Delta_{t} c \dot{\delta} t_{u, t} \\
c \dot{\delta} t_{u, t+1} & =\kappa c \dot{\delta} t_{u, t}+w_{c \dot{\delta} t_{u}, t}
\end{aligned}
$$

where $\kappa$ denotes the regression coefficient. By the vectormatrix form, we can also describe the model as follows (let call this model as the B-model of the receiver's clock error)

$$
\begin{aligned}
{\left[\begin{array}{c}
c \delta t_{u, t+1} \\
\dot{\delta} t_{u, t+1}
\end{array}\right] } & =\left[\begin{array}{cc}
1 & \Delta_{t} \\
0 & \kappa
\end{array}\right]\left[\begin{array}{c}
c \delta t_{u, t} \\
c \dot{\delta} t_{u, t}
\end{array}\right]+\left[\begin{array}{c}
0 \\
w_{c \dot{\delta} t_{u}, t}
\end{array}\right] \\
& \equiv F_{B, c \delta t}\left[\begin{array}{c}
c \delta t_{u, t} \\
c \dot{\delta} t_{u, t}
\end{array}\right]+\left[\begin{array}{c}
0 \\
w_{c \dot{\delta} t_{u}, t}
\end{array}\right]
\end{aligned}
$$

\subsection{Approximated Method of Estimation}

Now we present an approximated but simpler estimation algorithm of $\eta_{t}$. Namely define

$$
\eta_{t} \equiv\left[\begin{array}{c}
\eta_{1, t} \\
\vdots \\
\eta_{n_{r}, t}
\end{array}\right]
$$

where

$\eta_{i, t}=\left[\begin{array}{c}u_{i} \\ c \delta t_{u_{i}} \\ c \dot{\delta} t_{u_{i}} \\ \delta b_{u_{i}} \\ N_{L 1, u_{i}}\end{array}\right]:\left(6+n_{s}\right) \times 1, \quad i=1, \cdots, n_{r}$ 
Then the state equation and measurement equation are given by

$$
\begin{aligned}
\eta_{i, t+1} & =A_{i, t} \eta_{i, t}+w_{i, t}, \\
y_{\hat{u}_{i, t}^{(\nu)}} & =C_{i, t} \eta_{i, t}+v_{i, t},
\end{aligned}
$$

where

$$
\begin{aligned}
& A_{i, t} \equiv {\left[\begin{array}{llll}
I_{3 \times 3} & & & \\
& F_{*, c \delta t} & \\
& & I
\end{array}\right] } \\
& C_{i, t} \equiv\left[\begin{array}{ccccc}
G_{\hat{u}_{i}^{(\nu)}} & \mathbf{1} & \mathbf{0} & \mathbf{1} & \\
G_{\hat{u}_{i}^{(\nu)}} & \mathbf{1} & \mathbf{0} & \mathbf{1} & \lambda_{1} I
\end{array}\right] \\
& w_{i, t} \equiv\left[\begin{array}{c}
\mathbf{0}_{3} \\
* \\
w_{c \dot{\delta} t_{u i}} \\
\mathbf{0}_{2+n_{s i}}
\end{array}\right] .
\end{aligned}
$$

Thus, for each state $\eta_{i, t}$, we can obtain the filtering estimate $\hat{\eta}_{i, t \mid t}$ and its error covariance matrix $\Sigma_{i, t \mid t}$ by applying the Kalman filter. The approximated estimate $\hat{\eta}_{t \mid t}$ and its error covariance are obtained by

$$
\hat{\eta}_{t \mid t}=\left[\begin{array}{c}
\hat{\eta}_{1, t \mid t} \\
\vdots \\
\hat{\eta}_{n_{r}, t \mid t}
\end{array}\right],
$$

and

$$
R_{\eta, t \mid t}=\left[\begin{array}{ccc}
\Sigma_{1, t \mid t} & & O \\
& \ddots & \\
O & & \Sigma_{2, t \mid t}
\end{array}\right]
$$

\subsection{Updating by the Constraints for PPP}

The constraint conditions are applied to update PPP estimates as follows. Namely, when we have obtained the filtering estimates $\hat{\eta}_{t \mid t}$ and the error covariance matrix $R_{t \mid t}$, we apply the constraint conditions:

$$
d_{j i, t} \approx \kappa_{j i}^{\mathrm{T}}\left(u_{j, t}-u_{i, t}\right)
$$

where

$$
\kappa_{j i} \equiv \frac{\left(\hat{u}_{j}^{(\nu)}-\hat{u}_{i}^{(\nu)}\right)}{\left\|\hat{u}_{j}^{(\nu)}-\hat{u}_{i}^{(\nu)}\right\|}
$$

Equivalently, we assume

$$
\begin{aligned}
d_{j i} & =\kappa_{j i}^{\mathrm{T}}\left(u_{j}-u_{i}\right)+e_{d_{j i}} \\
& \equiv \kappa_{j i}^{\mathrm{T}} u_{j i}+e_{d_{j i}}
\end{aligned}
$$

where $e_{d_{j i}}$ and $e_{c \delta_{j i}}$ are mutually independent Gaussian white noises with

$$
e_{d_{j i}} \sim \mathrm{N}\left(0, r_{d_{j i}}\right)
$$

Define

$d_{n_{r}, t} \equiv\left[d_{21, t}, d_{31, t}, d_{32, t}, d_{41, t}, d_{42, t}, \cdots \cdots, d_{n_{r} n_{r-1}, t}\right]^{\mathrm{T}}$,

then consider the following relations of the conditional probability density function (CPDF)

$$
\begin{aligned}
& p\left(\eta_{t} \mid Y^{t}, d_{n_{r}, t}\right) \\
= & \frac{p\left(\eta_{t}, Y^{t}, d_{n_{r}, t}\right)}{p\left(Y^{t}, d_{n_{r}, t}\right)}=\frac{p\left(\eta_{t}, d_{n_{r}, t} \mid Y^{t}\right) p\left(Y^{t}\right)}{p\left(Y^{t}, d_{n_{r}, t}\right)} \\
\equiv & \frac{p\left(d_{n_{r}, t} \mid \eta_{t}, Y^{t}\right) p\left(\eta_{t} \mid Y^{t}\right) p\left(Y^{t}\right)}{p\left(Y^{t}, d_{n_{r}, t}\right)} \\
= & K_{0}\left(Y^{t}, d_{n_{r}, t}\right) p\left(\eta_{t} \mid Y^{t}\right) p\left(d_{n_{r}, t} \mid \eta_{t}\right) .
\end{aligned}
$$

Then we have relations:

$$
\begin{aligned}
& p\left(\eta_{t} \mid Y^{t}\right) \\
= & \frac{1}{(2 \pi)^{n^{\prime} / 2}\left|R_{\eta, t \mid t}\right|} \\
& \times \exp \left\{-\frac{1}{2}\left[\eta_{t}-\hat{\eta}_{t \mid t}\right]^{\mathrm{T}} R_{\eta, t \mid t}^{-1}\left[\eta_{t}-\hat{\eta}_{t \mid t}\right]\right\}, \quad \text { (46) } \\
& p\left(d_{n_{r}, t} \mid \eta_{t}\right) \\
= & \prod_{j>i}^{n_{r}}\left[\frac{1}{\sqrt{2 \pi r_{d_{j i}}}} \exp \left\{-\frac{\left[d_{j i}-\kappa_{j i}^{\mathrm{T}}\left(u_{j}-u_{i}\right)\right]^{2}}{2 r_{d_{j i}}}\right\}\right] .
\end{aligned}
$$

Therefore, $p\left(d_{n_{r}, t} \mid Y^{t}, \eta_{t}\right)$ in (45) is expressed as follows:

$$
\begin{aligned}
& p\left(\eta_{t} \mid Y^{t}, d_{n_{r}, t}\right) \\
= & K_{0}\left(Y^{t}, d_{n_{r}, t}\right) \frac{1}{(2 \pi)^{n^{\prime} / 2}\left|R_{\eta, t \mid t}\right|} \\
& \times \exp \left\{-\frac{1}{2}\left[\eta_{t}-\hat{\eta}_{t \mid t}\right]^{\mathrm{T}} R_{\eta, t \mid t}^{-1}\left[\eta_{t}-\hat{\eta}_{t \mid t}\right]\right\} \\
& \times \prod_{j>i}^{n_{r}}\left[\frac{1}{\sqrt{2 \pi r_{d_{j i}}}} \exp \left\{-\frac{\left[d_{j i}-\kappa_{j i}^{\mathrm{T}}\left(u_{j}-u_{i}\right)\right]^{2}}{2 r_{d_{j i}}}\right\}\right] .
\end{aligned}
$$

Then the constraints appeared in the power forms of the exponential function are expressed by the quadratic form of $\eta$ as follows:

$$
\begin{aligned}
q_{j i} \equiv & \frac{\left[d_{j i}-\kappa_{j i}^{\mathrm{T}}\left(u_{j}-u_{i}\right)\right]^{2}}{2 r_{u_{j i}}} \\
= & \frac{1}{2 r_{u_{j i}}}\left\{d_{j i}^{2}+\kappa_{j i}^{\mathrm{T}}\left(u_{j}-u_{i}\right)\left(u_{j}-u_{i}\right)^{\mathrm{T}} \kappa_{j i}\right. \\
& \left.-2 d_{j i} \kappa_{j i}^{\mathrm{T}}\left(u_{j}-u_{i}\right)\right\} \\
= & \frac{1}{2 r_{u_{j i}}}\left\{d_{j i}^{2}+\left(u_{j}-u_{i}\right)^{\mathrm{T}} \kappa_{j i} \kappa_{j i}^{\mathrm{T}}\left(u_{j}-u_{i}\right)\right. \\
& \left.-2 d_{j i} \kappa_{j i}^{\mathrm{T}} u_{j}+2 d_{j i} \kappa_{j i}^{\mathrm{T}} u_{i}\right\} \\
= & \frac{1}{2 r_{u_{j i}}}\left\{d_{j i}^{2}+u_{j}^{\mathrm{T}} \kappa_{j i} \kappa_{j i}^{\mathrm{T}} u_{j}-u_{j}^{\mathrm{T}} \kappa_{j i} \kappa_{j i}^{\mathrm{T}} u_{i}-u_{i}^{\mathrm{T}} \kappa_{j i} \kappa_{j i}^{\mathrm{T}} u_{j}\right. \\
& \left.+u_{i}^{\mathrm{T}} \kappa_{j i} \kappa_{j i}^{\mathrm{T}} u_{i}-2 d_{j i} \kappa_{j i}^{\mathrm{T}} u_{j}+2 d_{j i} \kappa_{j i}^{\mathrm{T}} u_{i}\right\} \\
= & \frac{1}{2}\left\{\frac{d_{j i}^{2}}{r_{u_{j i}}}+u_{j}^{\mathrm{T}} K_{j i} u_{j}-u_{j}^{\mathrm{T}} K_{j i} u_{i}-u_{i}^{\mathrm{T}} K_{j i} u_{j}\right. \\
& \left.+u_{i}^{\mathrm{T}} K_{j i} u_{i}-d_{\kappa j i}^{\mathrm{T}} u_{j}+d_{\kappa j i}^{\mathrm{T}} u_{i}\right\},
\end{aligned}
$$


where

$$
\begin{aligned}
K_{j i} & \equiv \frac{1}{r_{u_{j i}}} \kappa_{j i} \kappa_{j i}^{\mathrm{T}} \quad(: 3 \times 3), \\
d_{\kappa j i}^{\mathrm{T}} & \equiv \frac{2 d_{j i} \kappa_{j i}^{\mathrm{T}}}{r_{u_{j i}}}(: 1 \times 3) .
\end{aligned}
$$

Therefore, in the case of $n_{r}=2$ (See the formulas for $n_{r}=3$ and 4 in [1]-[2], we have

$$
\begin{aligned}
& \frac{1}{2} \sum_{j>i}^{2}\left[\frac{\left[d_{j i}-\kappa_{j i}^{\mathrm{T}}\left(u_{i}-u_{j}\right)\right]^{2}}{r_{u_{j i}}}\right] \\
= & \frac{1}{2} q_{21} \\
= & \frac{1}{2}\left\{\frac{d_{21}^{2}}{r_{u_{21}}}+u_{2}^{\mathrm{T}} K_{21} u_{2}-u_{2}^{\mathrm{T}} K_{21} u_{1}-u_{1}^{\mathrm{T}} K_{21} u_{2}\right. \\
& \left.+u_{1}^{\mathrm{T}} K_{21} u_{1}-d_{\kappa 21}^{\mathrm{T}} u_{2}+d_{\kappa 21}^{\mathrm{T}} u_{1}\right\} \\
\equiv & \frac{1}{2}\left[\eta^{\mathrm{T}} M_{21} \eta+c_{21}^{\mathrm{T}} \eta+d_{r 21}\right.
\end{aligned}
$$

where

$$
\begin{aligned}
K_{21} & \equiv \frac{1}{r_{u_{21}}} \kappa_{21} \kappa_{21}^{\mathrm{T}} \quad(: 3 \times 3), \\
d_{\kappa 12}^{\mathrm{T}} & \equiv \frac{2 d_{12} \kappa_{12}^{\mathrm{T}}}{r_{u_{12}}} \quad(: 1 \times 3),
\end{aligned}
$$

and

$$
M_{21} \equiv\left[\begin{array}{cc|cc}
K_{21} & & -K_{21} & \\
& O_{n_{s}+3} & & O_{n_{s}+3} \\
\hline-K_{21} & O_{n_{s}+3} & & K_{21} \\
& & O_{n_{s}+3}
\end{array}\right]
$$

and

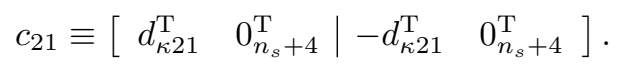

where $O_{n_{s}+3}$ and $0_{n_{s}+3}$ denote the $\left(n_{s}+3\right) \times\left(n_{s}+3\right)$ zero matrix and the $\left(n_{s}+3\right) \times 1$ zero vector, respectively. Therefore, finally we have the following quadratic form as the power term of (48)

$$
\begin{aligned}
- & \frac{1}{2}(\eta-\hat{\eta}) R^{-1}(\eta-\hat{\eta})-\frac{1}{2} q_{21} \\
=- & \frac{1}{2}\left\{\eta^{\mathrm{T}} R^{-1} \eta-\eta^{\mathrm{T}} R^{-1} \hat{\eta}-\hat{\eta}^{\mathrm{T}} R^{-1} \eta\right. \\
& \left.+\hat{\eta}^{\mathrm{T}} R^{-1} \hat{\eta}+\eta^{\mathrm{T}} M_{21} \eta+c_{21}^{\mathrm{T}} \eta+d_{r 21}\right\} \\
=\quad & -\frac{1}{2}\left\{\left[\eta-\left(R^{-1}+M_{21}\right)^{-1}\left(R^{-1} \hat{\eta}-\frac{1}{2} c_{21}\right)\right]^{\mathrm{T}}\right. \\
\times & \left(R^{-1}+M_{21}\right) \\
\times & {\left[\eta-\left(R^{-1}+M_{21}\right)^{-1}\left(R^{-1} \hat{\eta}-\frac{1}{2} c_{21}\right)\right] } \\
- & \left(R^{-1} \hat{\eta}-\frac{1}{2} c_{21}\right)^{\mathrm{T}}\left(R^{-1}+M_{21}\right)^{-1}\left(R^{-1} \hat{\eta}-\frac{1}{2} c_{21}\right) \\
+ & \left.\hat{\eta}^{\mathrm{T}} R^{-1} \hat{\eta}+d_{r 21}\right\} .
\end{aligned}
$$

Then the updated estimates $\check{\eta}$ and the updated error covariance $\check{R}$ of $\hat{\eta}$ are given by

$$
\begin{aligned}
\check{\eta}_{21} & =\left(R^{-1}+M_{21}\right)^{-1}\left(R^{-1} \hat{\eta}-\frac{1}{2} c_{21}\right), \\
\check{R} & =\left(R^{-1}+M_{21}\right)^{-1} .
\end{aligned}
$$

Using multiple antennas, we will show the attitude estimation method by obtaining the baseline vector between two of any antennas $u_{1}, \cdots, u_{n_{r}}$.

Let us write the final results of the double difference measurement equations as follows [3].

$$
\begin{array}{lll} 
& \tilde{\rho}_{C A, \hat{u}_{j} \hat{u}_{i}}^{\hat{q} \hat{p}} \\
\equiv \quad & \rho_{C A, u_{j} u_{i}}^{q p}-\|\hat{u}-\hat{s}\|_{j i}^{p q}+\left(g_{\hat{u}_{j}}^{\hat{q} \hat{p}}\right)^{\mathrm{T}} \hat{u}_{j}-\left(g_{\hat{u}_{i}}^{\hat{q} \hat{p}}\right)^{\mathrm{T}} \hat{u}_{i} \\
\cong \quad & \left(g_{\hat{u}_{j}}^{\hat{q} \hat{p}}\right)^{\mathrm{T}} u_{j}-\left(g_{\hat{u}_{i}}^{\hat{q} \hat{p}}\right)^{\mathrm{T}} u_{i}+\left(g_{\hat{u}_{j}}^{\hat{q}}\right)^{\mathrm{T}} e_{s_{j}^{q}}-\left(g_{\hat{u}_{i}}^{\hat{q}}\right)^{\mathrm{T}} e_{s_{i}^{q}} \\
& -\left[\left(g_{\hat{u}_{j}}^{\hat{p}}\right)^{\mathrm{T}} e_{s_{j}^{p}}+\left(g_{\hat{u}_{i}}^{\hat{p}}\right)^{\mathrm{T}} e_{s_{i}^{p}}\right]+e_{C A, u_{j} u_{i}}^{q p} \\
& \tilde{\Phi}_{L 1, \hat{u}_{j} \hat{u}_{i}}^{\hat{q} \hat{p}} \\
\equiv \quad & \Phi_{L_{1}, u_{j} u_{i}}^{q p}-\|\hat{u}-\hat{s}\|_{j i}^{p q}+\left(g_{\hat{u}_{j}}^{\hat{q} \hat{p}}\right)^{\mathrm{T}} \hat{u}_{j}-\left(g_{\hat{u}_{i}}^{\hat{q} \hat{p}}\right)^{\mathrm{T}} \hat{u}_{i} \\
\cong \quad & \left(g_{\hat{u}_{j} \hat{p}}^{\hat{q}}\right)^{\mathrm{T}} u_{j}-\left(g_{\hat{u}_{i}}^{\hat{q} \hat{p}}\right)^{\mathrm{T}} u_{i}+\left(g_{\hat{u}_{j}}^{\hat{q}}\right)^{\mathrm{T}} e_{s_{j}^{q}}-\left(g_{\hat{u}_{i}}^{\hat{q}}\right)^{\mathrm{T}} e_{s_{i}^{q}} \\
& -\left[\left(g_{\hat{u}_{j}}^{\hat{p}}\right)^{\mathrm{T}} e_{s_{j}^{p}}-\left(g_{\hat{u}_{i}}^{\hat{p}}\right)^{\mathrm{T}} e_{s_{i}^{p}}\right] \\
& +\lambda_{1} N_{L_{1}, u_{j} u_{i}}^{q p}+\lambda_{1} \varepsilon_{L_{1}, u_{j} u_{i}}^{q p}
\end{array}
$$

where

$$
\begin{aligned}
\|\hat{u}-\hat{s}\|_{j i}^{p q} \equiv & \left\|\hat{u}_{j}-\hat{s}_{j}^{q}\right\|-\left\|\hat{u}_{i}-\hat{s}_{i}^{q}\right\| \\
& -\left[\left\|\hat{u}_{j}-\hat{s}_{j}^{p}\right\|-\left\|\hat{u}_{i}-\hat{s}_{i}^{p}\right\|\right] \\
g_{\hat{u}_{j}}^{\hat{q} \hat{p}} & \equiv g_{\hat{u}_{j}}^{\hat{q}}-g_{\hat{u}_{j}}^{\hat{p}}, g_{\hat{u}_{i}}^{\hat{q} \hat{p}} \equiv g_{\hat{u}_{i}}^{\hat{q}}-g_{\hat{u}_{i}}^{\hat{p}} \\
g_{\hat{u}_{j} \hat{u}_{i}}^{\hat{q}} & \equiv g_{\hat{u}_{j}}^{\hat{q}}-g_{\hat{u}_{i}}^{\hat{q}}, g_{\hat{u}_{j} \hat{u}_{i}}^{\hat{p}} \equiv g_{\hat{u}_{j}}^{\hat{p}}-g_{\hat{u}_{i}}^{\hat{p}} \\
e_{C A, u_{i}}^{q p} & \equiv e_{C A, u_{i}}^{q}-e_{C A, u_{i}}^{p} \\
e_{C A, u_{j}}^{q p} & \equiv e_{C A, u_{j}}^{q}-e_{C A, u_{j}}^{p} \\
e_{C A, u_{j} u_{i}}^{q p} & \equiv e_{C A, u_{j}}^{q p}-e_{C A, u_{i}}^{q p} .
\end{aligned}
$$

For the case of $p=1, q=2, \ldots, n_{s}$ and $u_{i}=u_{1}, u_{j}=u_{2}$ $\left(n_{r}=2\right)$, we have the following measurement equation for antennas of $u_{1}$ and $u_{2}$ and for $n_{s}$ satellites.

$$
y_{u_{2} u_{1}}^{n_{s}} \equiv C_{\hat{u}}^{n_{s}} \xi_{u_{2} u_{1}}^{n_{s}}+v_{u_{2} u_{1}}^{n_{s}}
$$

where

$$
\begin{aligned}
y_{u_{2} u_{1}}^{n_{s}} & \equiv\left[\begin{array}{c}
\tilde{\rho}_{C A, \hat{u}_{2} \hat{u}_{1}}^{\hat{n}_{s} \hat{1}} \\
\tilde{\Phi}_{L 1, \hat{u}_{2} \hat{u}_{1}}^{\hat{n}_{s} \hat{1}}
\end{array}\right], \xi_{u_{2} u_{1}}^{s 1}=\left[\begin{array}{c}
u_{1} \\
u_{2} \\
N_{L 1, u_{2} u_{1}}^{n_{s}}
\end{array}\right], \\
C_{\hat{u}}^{n_{s}} & =\left[\begin{array}{ccc}
-G_{\hat{u}_{1}}^{\hat{s} \hat{1}} & G_{\hat{u}_{2}}^{\hat{s} \hat{1}} & \boldsymbol{O} \\
-G_{\hat{u}_{1}}^{\hat{s} \hat{1}} & G_{\hat{u}_{2}}^{\hat{s} \hat{1}} & \lambda_{1} \boldsymbol{I}
\end{array}\right], \\
G_{u_{i}}^{\hat{s}_{i} \hat{1}_{i}} & \equiv\left[\begin{array}{lll}
\left(g_{\hat{u}_{1}}^{\hat{2} \hat{1}}\right) & \cdots & \left(g_{\hat{u}_{1}}^{\hat{n}_{s} \hat{1}}\right)
\end{array}\right]^{\mathrm{T}}:\left(n_{s}-1\right) \times 3 .
\end{aligned}
$$

where $y_{C A, \hat{u}_{2} \hat{u}_{1}}^{\hat{n_{s}}}$ and $\hat{y_{L 1} \hat{n_{s}} \hat{u}_{2} \hat{u}_{1}}$ are $\left(\left(n_{s}-1\right) \times 1\right)$ DD observables from raw C/A code pseudoranges and L1 carrier phases, respectively, and $v$ is the observation noise vector. 


\section{Update by Constraints for DD-VPPP}

The constraint conditions are applied to update DD-based baseline vector estimation as follows. In the case of $n_{r}=2$, define the baseline vector length constraints at time $t$ as follows:

$$
d_{21, t}=\left\|u_{2, t}-u_{1, t}\right\|+e_{d_{21, t}}=\left\|u_{21, t}\right\|+e_{d_{21, t}}
$$

where $e_{d_{21, t}}$ is a Gaussian white noise caused by the phase center variations of antennas with

$$
e_{d_{21, t}} \sim N\left(0, r_{d_{21, t}}\right)
$$

When we apply the constraint conditions to the filtering estimates $\hat{\xi}_{t \mid t}$ and the error covariance matrix $\Sigma_{\xi, t \mid t}$, we can consider the following relations of the conditional probability density function (CPDF):

$$
\begin{aligned}
p\left(\xi_{t} \mid Y^{t}, d_{21, t}\right) & =\frac{p\left(\xi_{t}, Y^{t}, d_{21, t}\right)}{p\left(Y^{t}, d_{21, t}\right)}=\frac{p\left(\xi_{t}, d_{21, t} \mid Y^{t}\right) p\left(Y^{t}\right)}{p\left(Y^{t}, d_{21, t}\right)} \\
& =\frac{p\left(d_{21, t} \mid \xi_{t}, Y^{t}\right) p\left(\xi_{t} \mid Y^{t}\right) p\left(Y^{t}\right)}{p\left(Y^{t}, d_{21, t}\right)} \\
& =K_{\xi}\left(Y^{t}, d_{21, t}\right) p\left(\xi_{t} \mid Y^{t}\right) p\left(d_{21, t} \mid \xi_{t}\right)
\end{aligned}
$$

Then we have relations:

$$
\begin{aligned}
& p\left(\xi_{t} \mid Y^{t}\right) \\
= & \frac{1}{(2 \pi)^{n^{\prime} / 2}\left|\Sigma_{\xi, t \mid t}\right|^{1 / 2}} \\
& \exp \left\{-\frac{1}{2}\left(\xi_{t}-\hat{\xi}_{t \mid t}\right)^{\mathrm{T}} \Sigma_{\xi, t \mid t}^{-1}\left(\xi_{t}-\hat{\xi}_{t \mid t}\right)\right\}
\end{aligned}
$$

And we have relations from Eq. (61):

$$
\begin{aligned}
& p\left(l_{21, t} \mid \xi_{t}\right) \\
= & \frac{1}{\sqrt{2 \pi r_{l_{21}}}} \exp \left\{-\frac{\left\{l_{21, t}-\left\|u_{21, t}\right\|\right\}^{2}}{2 r_{l_{21}}}\right\}
\end{aligned}
$$

Therefore, Eq. (63) is expressed as follows:

$$
\begin{aligned}
& p\left(\xi_{t} \mid Y^{t}, d_{21, t}\right) \\
= & K_{\xi}\left(Y^{t}, d_{21, t}\right) \\
& \times \frac{1}{(2 \pi)^{n^{\prime} / 2}\left|\Sigma_{\xi, t \mid t}\right|^{1 / 2}} \\
& \times \exp \left\{-\frac{1}{2}\left(\xi_{t}-\hat{\xi}_{t \mid t}\right)^{\mathrm{T}} \Sigma_{\xi, t \mid t}^{-1}\left(\xi_{t}-\hat{\xi}_{t \mid t}\right)\right\} \\
& \times \frac{1}{\sqrt{2 \pi r_{d_{21}}}} \exp \left\{-\frac{\left\{d_{21, t}-\left\|u_{21, t}\right\|\right\}^{2}}{2 r_{d_{21}}}\right\}
\end{aligned}
$$

Then we remark that the power term of $p\left(d_{21, t} \mid \xi_{t}\right)$ in Eq. (65) is expressed by the quadratic form of $\xi_{21}$ as follows:

$$
\begin{aligned}
& \frac{\left\{d_{21}-\left\|u_{21}\right\|\right\}^{2}}{2 r_{u_{21}}} \\
= & \frac{1}{2 r_{d_{21}}}\left(d_{21}^{2}+u_{21}^{\mathrm{T}} u_{21}-2 d_{21} \kappa_{21}^{\mathrm{T}} u_{21}\right) \\
= & \frac{1}{2}\left(u_{21}^{\mathrm{T}} \frac{I}{r_{d_{21}}} u_{21}-\frac{2 d_{21}}{r_{d_{21}}} \kappa_{21}^{\mathrm{T}} u_{21}+\frac{d_{21}^{2}}{r_{d_{21}}}\right) \\
= & \frac{1}{2}\left(\xi_{21}^{\mathrm{T}} M_{\xi_{21}} \xi_{21}-2 c_{\xi_{21}}^{\mathrm{T}} \xi_{21}+\frac{d_{21}^{2}}{r_{d_{21}}}\right),
\end{aligned}
$$

where

$$
\begin{aligned}
M_{\xi_{21}} & \equiv\left[\begin{array}{c|c}
K_{d_{21}} & O \\
\hline O & O_{n_{s}-1}
\end{array}\right], c_{\xi_{21}} \equiv\left[\begin{array}{ll}
c_{d_{21}}^{\mathrm{T}} & 0_{n_{s}-1}^{\mathrm{T}}
\end{array}\right] \\
K_{d_{21}} & \equiv \frac{I}{r_{d_{21}}} \quad(: 3 \times 3), \quad c_{d_{21}}^{\mathrm{T}} \equiv \frac{d_{21} \kappa_{21}^{\mathrm{T}}}{r_{d_{21}}}
\end{aligned}
$$

$O_{n_{s}-1}$ is $\left(n_{s}-1\right) \times\left(n_{s}-1\right)$ zero matrix, and $0_{n_{s}-1}$ is $1 \times\left(n_{s}-1\right)$ zero vector. Therefore, finally we have the following quadratic form for the power term of the CPDF (66):

$$
\begin{aligned}
& \frac{1}{2}(\xi-\hat{\xi}) \Sigma^{-1}(\xi-\hat{\xi})+\frac{1}{2} \frac{\left\{d_{21}-\left\|u_{21}\right\|\right\}^{2}}{r_{d_{21}}} \\
\cong \quad \frac{1}{2}\left[(\xi-\hat{\xi}) \Sigma^{-1}(\xi-\hat{\xi})\right. & \\
& \left.\left.+\xi_{21}^{\mathrm{T}} M_{\xi_{21}} \xi_{21}-2 c_{\xi_{21}}^{\mathrm{T}} \xi_{21}+\frac{d_{21}^{2}}{r_{d_{21}}}\right)\right] \\
=\quad & \frac{1}{2}\left\{\xi^{\mathrm{T}} \Sigma^{-1} \xi-\xi^{\mathrm{T}} \Sigma^{-1} \hat{\xi}-\hat{\xi}^{\mathrm{T}} \Sigma^{-1} \xi\right. \\
& \left.+\hat{\xi}^{\mathrm{T}} \Sigma^{-1} \hat{\xi}+\xi^{\mathrm{T}} M_{\xi_{21}} \xi-2 c_{\xi_{21}}^{\mathrm{T}} \xi+\frac{d_{21}^{2}}{r_{d_{21}}}\right\} \\
= & \frac{1}{2}\left\{\xi^{\mathrm{T}}\left(\Sigma^{-1}+M_{\xi_{21}}\right) \xi-\xi^{\mathrm{T}}\left(\Sigma^{-1} \hat{\xi}+c_{\xi_{21}}\right)\right. \\
& \left.-\left(\hat{\xi}^{\mathrm{T}} \Sigma^{-1}+c_{\xi_{21}}^{\mathrm{T}}\right) \xi+\hat{\xi}^{\mathrm{T}} \Sigma^{-1} \hat{\xi}+\frac{d_{21}^{2}}{r_{d_{21}}}\right\} \\
& \frac{1}{2}\left\{\left[\xi-\left(\Sigma^{-1}+M_{\xi_{21}}\right)^{-1}\left(\Sigma^{-1} \hat{\xi}+c_{\xi_{21}}\right)\right]^{\mathrm{T}}\left(\Sigma^{-1}+M_{\xi_{21}}\right)\right. \\
\times & {\left[\xi-\left(\Sigma^{-1}+M_{\xi_{21}}\right)^{-1}\left(\Sigma^{-1} \hat{\xi}+c_{\xi_{21}}\right)\right] } \\
- & \left(\Sigma^{-1} \hat{\xi}+c_{\xi_{21}}\right)^{\mathrm{T}}\left(\Sigma^{-1}+M_{\xi_{21}}\right)^{-1}\left(\Sigma^{-1} \hat{\xi}+c_{\xi_{21}}\right) \\
+ & \left.\hat{\xi}^{\mathrm{T}} \Sigma^{-1} \hat{\xi}+\frac{d_{21}^{2}}{r_{d_{21}}}\right\} .
\end{aligned}
$$

Then the update estimated vector $\check{\xi}_{21}$ and error covariance matrix $\check{\Sigma}_{\xi_{21}}$ of $\xi_{21}$ based on the minimum mean square estimate are given by

$$
\begin{aligned}
\check{\xi}_{21} & =\left(\Sigma_{\xi_{21}}^{-1}+M_{\xi_{21}}\right)^{-1}\left(\Sigma_{\xi_{21}}^{-1} \hat{\xi}_{21}+c_{\xi_{21}}\right) \\
\check{\Sigma}_{\xi_{21}} & =\left(\Sigma_{\xi_{21}}^{-1}+M_{\xi_{21}}\right)^{-1} .
\end{aligned}
$$




\section{Determination of Integer Ambiguity [4]}

Based on the Kalman filtering and the constraint conditions, we obtain the estimates

$$
\hat{u}, \quad\left(\hat{u} \equiv\left[\begin{array}{ll}
\hat{u}_{1}^{\mathrm{T}} & \hat{u}_{2}^{\mathrm{T}}
\end{array}\right]^{\mathrm{T}}, \quad \hat{N}\right.
$$

and the error covariance

$$
\Sigma_{\xi}=\operatorname{cov}\left\{\left[\begin{array}{c}
u-\hat{u} \\
N-\hat{N}
\end{array}\right]\right\}=\left[\begin{array}{cc}
\Sigma_{\hat{u}} & \Sigma_{\hat{u} \hat{N}} \\
\Sigma_{\hat{N} \hat{u}} & \Sigma_{\hat{N}}
\end{array}\right]
$$

Then once we get the integer ambiguity $N$, we can update the estimate $\hat{u}$ to $\check{u}(N)$ as follow:

$$
\check{u}(N)=\hat{u}+\Sigma_{\hat{u} \hat{N}} \Sigma_{\hat{N}}^{-1}\left(N_{c}-\hat{N}\right)
$$

Then we have the relation:

$$
\begin{aligned}
d_{21} & =\left\|\check{u}_{2}(N)-\check{u}_{1}(N)\right\|+e_{\tilde{d}_{21}}, e_{\tilde{d}_{21}} \sim N\left(0, r_{\tilde{d}_{21}}\right) \\
& =\left\|\check{u}_{21}(N)\right\|+e_{\tilde{d}_{21}}
\end{aligned}
$$

Likelihood function of integer ambiguity $N$ with constrains

The likelihood function of $\mathrm{N}$ is given by $L(N) \equiv$ $p\left(\hat{N}, \tilde{d}_{21} \mid N\right)$. Then we have the maximum likelihood estimate $\check{N}$ of $N$ as follows:

$$
\begin{aligned}
\check{N} & =\underset{N \in Z^{m}}{\arg \max } p\left(\hat{N}, d_{21} \mid N\right) \\
& =\underset{N \in Z^{m}}{\arg \max } p(\hat{N} \mid N) p\left(d_{21} \mid \hat{N}, N\right)
\end{aligned}
$$

where

$$
\begin{aligned}
& p\left(d_{21} \mid \hat{N}, N\right) \\
= & \frac{1}{\sqrt{2 \pi r_{\tilde{d}_{21}}}} \exp \left\{-\frac{\left[\tilde{d}_{21}-\| \check{u}_{21}(N)||\right]^{2}}{2 r_{d_{21}}}\right\} \\
& p(\hat{N} \mid N) \\
= & \frac{1}{(2 \pi)^{n / 2}\left|\Sigma_{\hat{N}}\right|^{1 / 2}} \exp \left\{-\frac{1}{2}\|\hat{N}-N\|_{\Sigma_{\hat{N}}^{-1}}\right\}
\end{aligned}
$$

From (74), (75), (76), we have

$$
\begin{aligned}
\check{N} & =\underset{N \in Z^{m}}{\arg \max } p\left(\hat{N}, d_{21} \mid N\right) \\
& =\underset{N \in Z^{m}}{\arg \min }\left\{\|\hat{N}-N\|_{\Sigma_{\hat{N}}^{-1}}^{2}+\frac{\left(d_{21}-\left\|\check{u}_{21}(N)\right\|\right)^{2}}{r_{d_{21}}}\right\}
\end{aligned}
$$

Practically, for obtaining $\check{N}$ in (77), we define the set of integers; $N_{c}$ which is obtained by applying the LAMBDA method [9]:

$$
N_{c} \equiv\left\{N_{c, 1}, N_{c, 2}, \cdots, N_{c, n}\right\}
$$

Then we can obtain

$$
\check{N}=\underset{N \in N_{c, i}}{\arg \min }\left\{\|\hat{N}-N\|_{\Sigma_{\hat{N}}^{-1}}^{2}+\frac{\left(d_{21}-\left\|\check{u}_{21}(N)\right\|\right)^{2}}{r_{d_{21}}}\right\}
$$

Then we again update the estimate the antennas' positions as follow:

$$
\check{u}=\check{u}(\check{N})=\hat{u}+\Sigma_{\hat{u} \hat{N}} \Sigma_{\hat{N}}^{-1}(\check{N}-\hat{N})
$$

\section{Euler angle estimation by parameters of Euler's principal rotation theorem}

The rotation angles $\alpha, \beta, \gamma$ around X,Y,Z-axes, respectively, are called Euler angles, and the corresponding rotation matrixes are as follows:

$$
\begin{aligned}
& \Gamma(X, \alpha) \equiv\left[\begin{array}{ccc}
1 & 0 & 0 \\
0 & \cos \alpha & \sin \alpha \\
0 & -\sin \alpha & \cos \alpha
\end{array}\right] \\
& \Gamma(Y, \beta) \equiv\left[\begin{array}{ccc}
\cos \beta & 0 & -\sin \beta \\
0 & 1 & 0 \\
\sin \beta & 0 & \cos \beta
\end{array}\right] \\
& \Gamma(Z, \gamma) \equiv\left[\begin{array}{ccc}
\cos \gamma & \sin \gamma & 0 \\
-\sin \gamma & \cos \gamma & 0 \\
0 & 0 & 1
\end{array}\right]
\end{aligned}
$$

In the case of $n_{r}=2$, the rotation matrix of Z-Y-X sequence from the vector $r_{f, N, 21}$ on the body coordinates which are reference coordinates to the estimated baseline vector $\hat{u}_{N, 21}$ on the navigation coordinates is as follows:

$$
\begin{aligned}
\hat{u}_{N, 21} & =\Gamma(X, \alpha) \Gamma(Y, \beta) \Gamma(Z, \gamma) r_{f, N, 21} \\
& =\Gamma(\alpha, \beta, \gamma) r_{f, N, 21},
\end{aligned}
$$

where $\mathrm{N}$ denotes normalized,

$$
\begin{aligned}
r_{f, N, 21} & \equiv \frac{r_{f, 21}}{\left\|r_{f, 21}\right\|}=\left[x_{N, 21}^{(R)}, y_{N, 21}^{(R)}, z_{N, 21}^{(R)}\right]^{\mathrm{T}} \\
\hat{u}_{N, 21} & \equiv \frac{\hat{u}_{21}}{\left\|\hat{u}_{21}\right\|}=\left[\hat{x}_{N, 21}, \hat{y}_{N, 21}, \hat{z}_{N, 21}\right]^{\mathrm{T}} \\
\hat{u}_{21} & \equiv \hat{u}_{2}-\hat{u}_{1}
\end{aligned}
$$

The composition of rotation matrix $\Gamma(\alpha, \beta, \gamma)$ in Eq. (81) is expressed by the parameters based on Euler's principal rotation theorem as follows [10]:

$$
\begin{aligned}
& \Gamma(\alpha, \beta, \gamma)=\tilde{\Gamma}(\phi, \mathbf{n}) \\
= & {\left[\begin{array}{ccc}
e+h n_{x}{ }^{2} & h n_{x} n_{y}-w n_{z} & h n_{z} n_{x}+w n_{y} \\
h n_{x} n_{y}+w n_{z} & e+h n_{y}{ }^{2} & h n_{y} n_{z}-w n_{x} \\
h n_{z} n_{x}-w n_{y} & h n_{y} n_{z}+w n_{x} & e+h n_{z}{ }^{2}
\end{array}\right] }
\end{aligned}
$$

where $\phi$ is the angle between two vectors $r_{f, N, 21}$ and $\hat{u}_{N, 21}$, and $\mathbf{n}$ is the common normal vector of two vector $r_{f, N, 21}$ 
and $\hat{u}_{N, 21}$. The vector $r_{f, N, 21}$ is identical with the vector $\hat{u}_{N, 21}$ by rotating of the angle $\phi$ around the principal axis $\mathbf{n}$,

$$
\begin{aligned}
& \mathbf{n}=\left(n_{x}, n_{y}, n_{z}\right)^{\mathrm{T}}=\frac{r_{f, N, 21} \times \hat{u}_{N, 21}}{\left\|r_{f, N, 21} \times \hat{u}_{N, 21}\right\|} \\
& e=r_{f, N, 21} \cdot \hat{u}_{N, 21}=\cos \phi \quad: \text { outer product } \\
& h=e-1, w=\sqrt{1-e^{2}}=\sin \phi .
\end{aligned}
$$

In the case of multiple antennas, we derive the computational algorithms by estimating the multiple baseline vectors $\hat{u}_{N, j i}$ from the reference antenna $i$ to other antennas $j$ disposed in a plane [10]. The appropriate rotation matrix $\Gamma$ to minimize the rotation errors from the multiple reference baseline vectors $r_{f, N, j i}$ to the multiple baseline vectors $\hat{u}_{N, j i}$ is estimated by the least square (LS) method, and that is the corresponding equations to estimate the parameters $\theta$ Euler's principal rotation theorem as follows (See Appendix):

$$
\min _{\Gamma} \sum_{i<j}^{n_{r}}\left\|\hat{u}_{N, j i}-\Gamma r_{f, N, j i}\right\|^{2}=\min _{\theta} \sum_{i<j}^{n_{r}}\left\|\hat{u}_{N, j i}-H_{j i} \theta\right\|^{2}
$$

where

$$
\begin{aligned}
\hat{u}_{N . j i} & \equiv\left[\begin{array}{c}
\hat{x}_{N, j i} \\
\hat{y}_{N, j i} \\
\hat{z}_{N, j i}
\end{array}\right], \theta \equiv\left[\begin{array}{c}
\cos \phi \\
n_{x} \sin \phi \\
n_{y} \sin \phi \\
n_{z} \sin \phi
\end{array}\right], \\
H_{j i} & \equiv\left[\begin{array}{cccc}
x_{N, j i}^{(R)} & 0 & z_{N, j i}^{(R)} & -y_{N, j i}^{(R)} \\
y_{N, j i}^{(R)} & -z_{N, j i}^{(R)} & 0 & x_{N, j i}^{(R)} \\
z_{N, j i}^{(R)} & y_{N, j i}^{(R)} & -x_{N, j i}^{(R)} & 0
\end{array}\right] .
\end{aligned}
$$

The Euler angle estimation by the parameters of Euler's principal rotation theorem under Z-Y-X sequence is as follows. First of all, the multiple vectors $r_{f, N, j i}$ and $\hat{u}_{j i}$ are normalized as shown in (85), and mapped on the X-Y,X$\mathrm{Z}$, and $\mathrm{Y}-\mathrm{Z}$ planes to estimate the rotation angles in the sequence. Then the parameters $\hat{\theta}(Z, \gamma), \hat{\theta}(Y, \beta)$, and $\hat{\theta}(X, \alpha)$ for the rotations around $\mathrm{Z}$-axis, $\mathrm{Y}$-axis and $\mathrm{X}$-axis, respectively is estimated by the LS method in the sequence as follows:

$$
\hat{\theta}=\left(\sum_{i<j}^{n_{r}}\left(H_{j i}^{\mathrm{T}} H_{j i}\right)\right)^{-1} \sum_{i<j}^{n_{r}}\left(H_{j i}^{\mathrm{T}} \hat{u}_{N, j i}\right),
$$

and each rotation matrix $\Gamma(Z, \gamma), \Gamma(Y, \beta)$, and $\Gamma(X, \alpha)$ in (86), respectively, is derived through (89) from the parameter $\hat{\theta}$. Finally, the Euler angles are derived from each rotation matrix in the sequence as follows:

$$
\alpha=\tan ^{-1} \frac{\Gamma_{32}}{\Gamma_{33}}, \beta=\tan ^{-1} \frac{-\Gamma_{31}}{\sqrt{\Gamma_{32}^{2}+\Gamma_{33}^{2}}}, \gamma=\tan ^{-1} \frac{\Gamma_{21}}{\Gamma_{11}}
$$

where $\Gamma_{21}$ denotes the matrix element of the second row and first column.

\section{EXPERIMENTAL RESULTS}

\subsection{Positioning Experiment}

We have carried out the experiments of using the GPS data

\begin{tabular}{|c|c|}
\hline $\begin{array}{c}\text { Date } \\
\text { (GPS-Time) }\end{array}$ & $\begin{array}{c}\text { March 8, 2015 } \\
\left(06: 055^{\prime} 00 \sim 06: 29{ }^{\prime} 59\right)\end{array}$ \\
\hline Location & BK Campus, Ritsumeikan Univ. \\
\hline Antenna(Ant) & u-blox patch antenna for automobile \\
\hline Receiver & u-blox GPS-module NEO-M8N \\
\hline Epoch interval & $1[\mathrm{~s}]$ \\
\hline Elevation angle mask & 5 [deg.] \\
\hline Measurement Data & rier-phase \\
\hline
\end{tabular}
(see Table 1) from the antennas Ant.1,2 located at the Fig. 1, and the coordinates of their reference positions in the WGS84 system are listed in Table 2 which are obtained by applying the relative positioning method. The positioning error applying the relative positioning method is less than a few $\mathrm{cm}$.

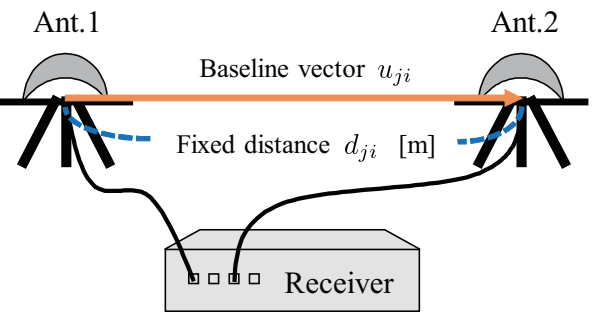

Fig. 1: Antennas in Experiment

Table 1: Experimental Conditions

Table 2: Antennas' positions

\begin{tabular}{|c|c|c|c|}
\hline & \multicolumn{3}{|c|}{ WGS-84 } \\
\cline { 2 - 4 } & $\mathrm{X}[\mathrm{m}]$ & $\mathrm{Y}[\mathrm{m}]$ & $\mathrm{Z}[\mathrm{m}]$ \\
\hline \hline Ant.1 & -3761236.152 & 3636879.281 & 3635962.036 \\
\hline Ant.2 & -3761236.392 & 3636878.384 & 3635962.676 \\
\hline
\end{tabular}

We have carried out experiments to compare the estimation accuracy of Broadcast Ephmeris and MADOCA Products. 


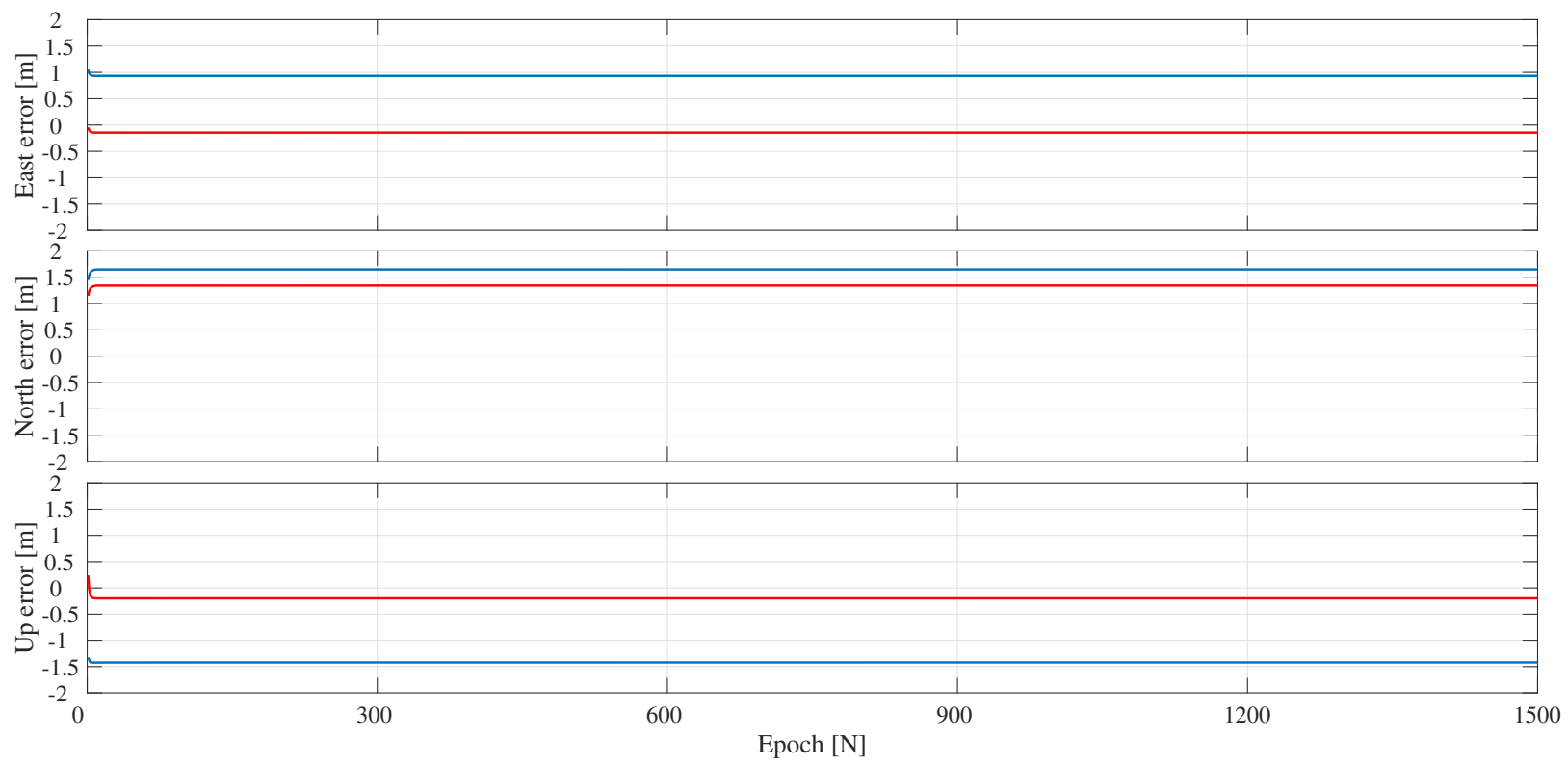

Fig. 2: Positioning Errors at Ant.1

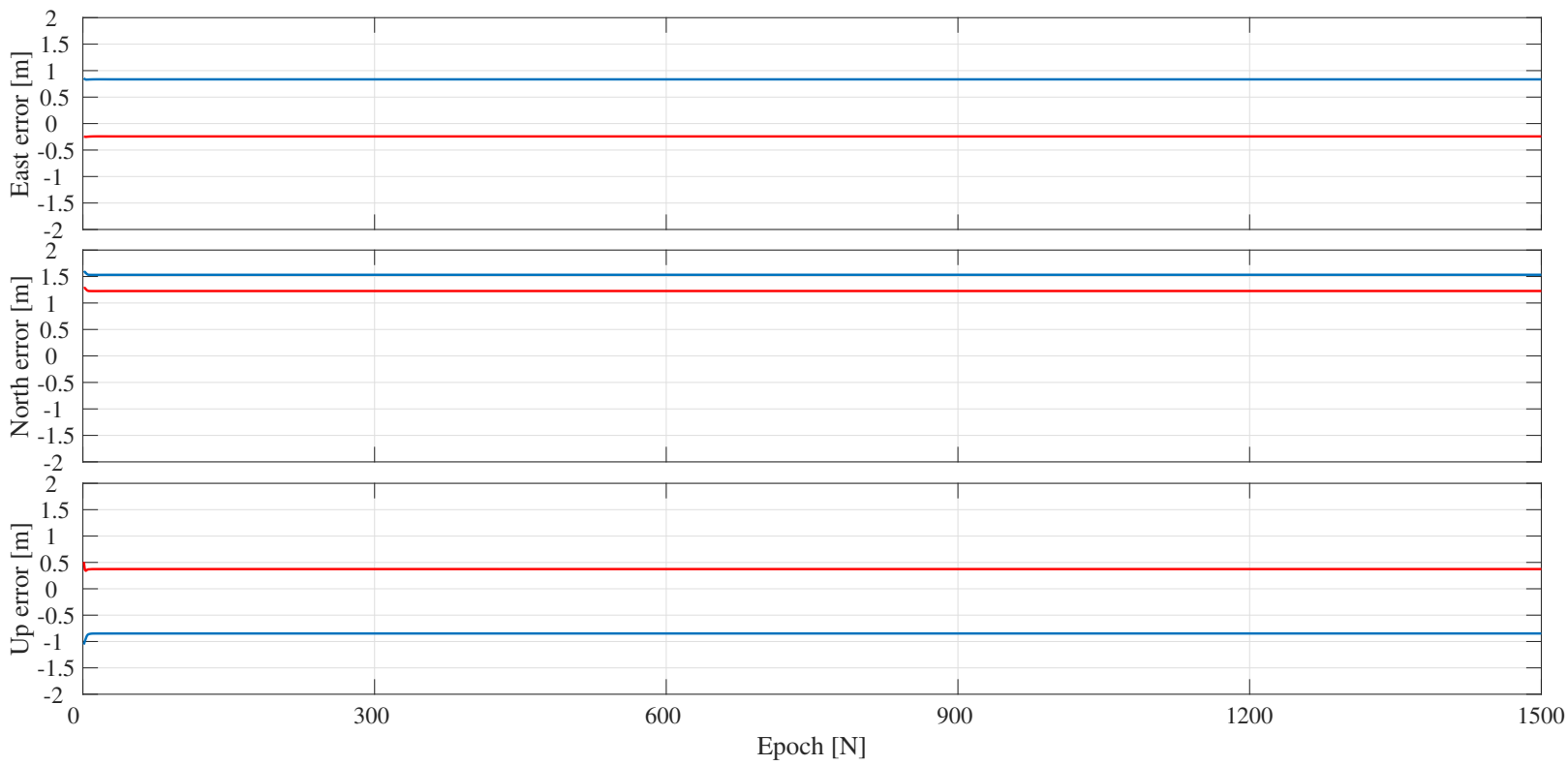

Fig. 3: Positioning Errors at Ant.2

Table 3 : RMS-ERRORS

\begin{tabular}{|c|c|c|c|c|}
\hline & & & $\mathrm{RM}$ & {$[\mathrm{m}]$} \\
\hline \multirow{6}{*}{ Ant.1 } & \multirow{3}{*}{ Broadcast Ephemeris } & $\mathrm{E}$ & 0.9333 & \multirow{3}{*}{1.3666} \\
\hline & & $\mathrm{N}$ & 1.6477 & \\
\hline & & $\mathrm{U}$ & 1.4201 & \\
\hline & \multirow{3}{*}{ MADOCA Products } & $\mathrm{E}$ & 0.1442 & \multirow{3}{*}{0.7874} \\
\hline & & $\mathrm{N}$ & 1.3415 & \\
\hline & & $\mathrm{U}$ & 0.1989 & \\
\hline \multirow{6}{*}{ Ant. 2} & \multirow{3}{*}{ Broadcast Ephemeris } & $\mathrm{E}$ & 0.8348 & \multirow{3}{*}{1.1204} \\
\hline & & $\mathrm{N}$ & 1.5328 & \\
\hline & & $\mathrm{U}$ & 0.8482 & \\
\hline & \multirow{3}{*}{ MADOCA Products } & $\mathrm{E}$ & 0.2427 & \multirow{3}{*}{0.7534} \\
\hline & & $\mathrm{N}$ & 1.2266 & \\
\hline & & $\bar{U}$ & 0.3734 & \\
\hline
\end{tabular}

Figs.2,3 shows the positioning errors with the local level axes (East, North and Upper), where the dotted blue lines show the errors for using the Broadcast Ephemeris and the solid red lines show the errors for using MADOCA Products.

The errors were computed by difference between the estimated positions and the coresponding positions in the co- 
ordinates of Table 3. We can observe from Figs. 2, 3 that the positioning quality is considerably improved by using MADOCA Products.

The statistics of the positioning results are summarized in Table 3, where RMS errors are shown for using the Broadcast Ephemeris and MADOCA Products respectively for two antennas.

\subsection{Baseline Vector Estimations Experiment}

We estimated baseline vector $u_{21}$ existing between two antennas (Ant.1, Ant.2) located at the Fig. 1.

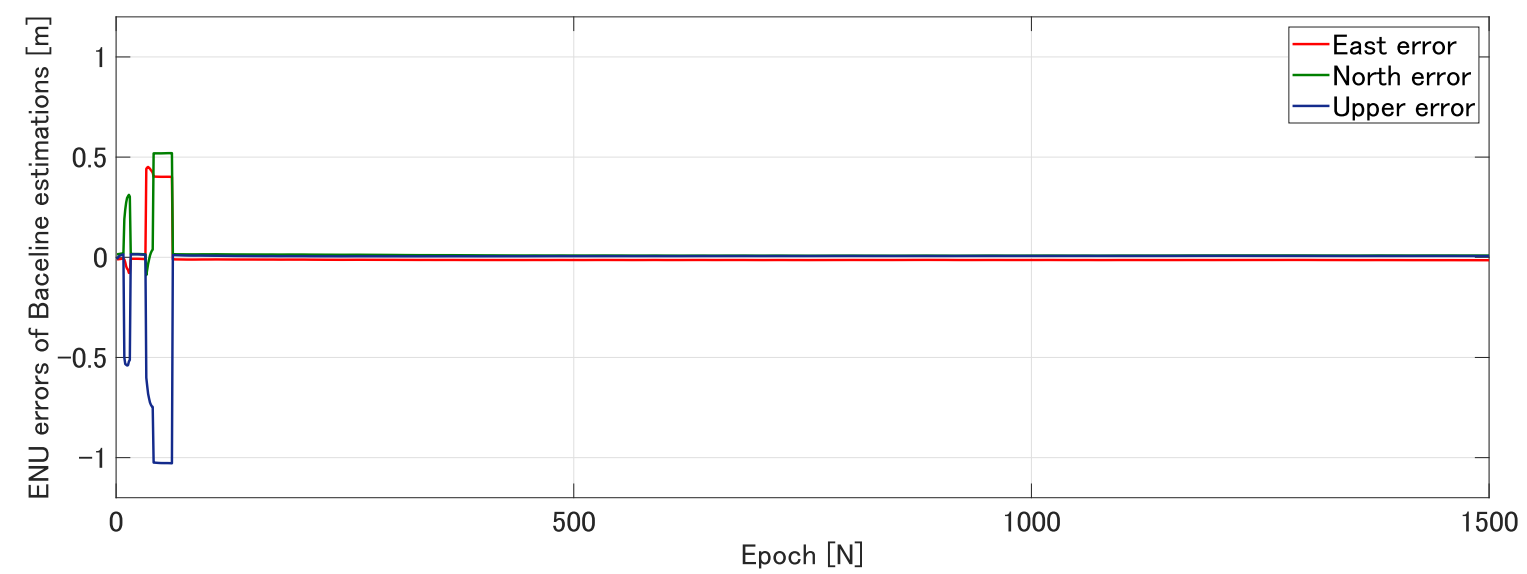

Fig. 4: ENU errors of baseline estimations [Broadcast Ephmeris]

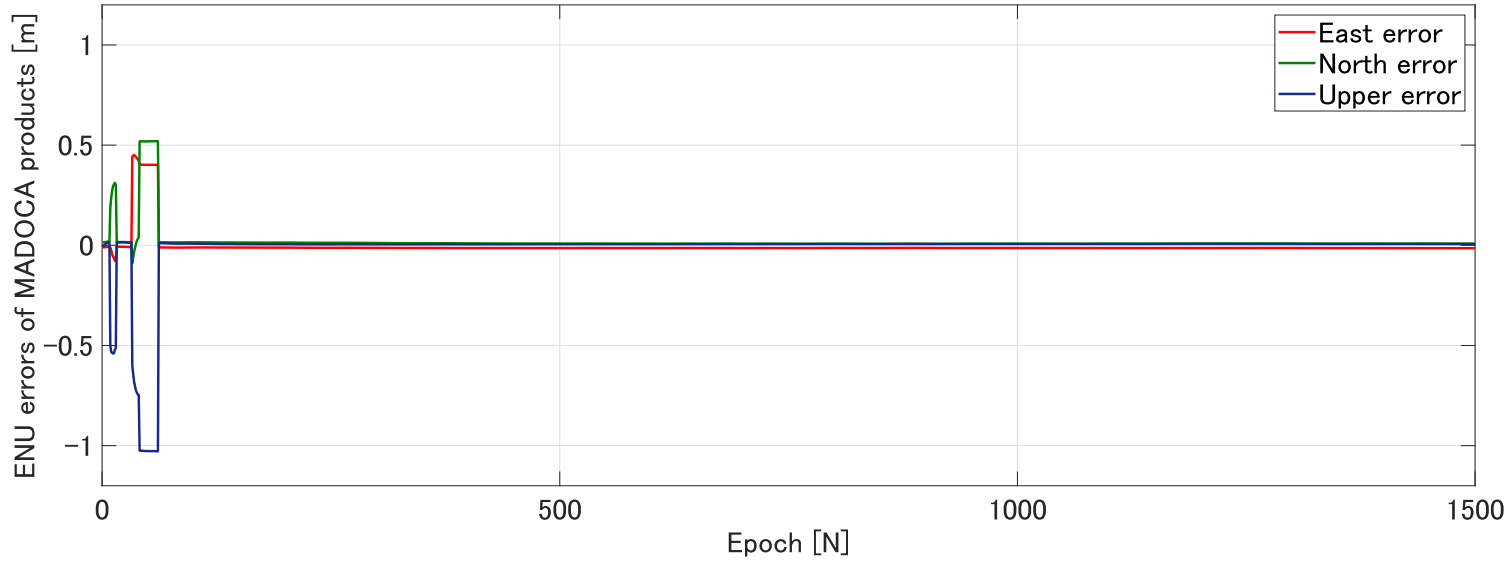

Fig. 5: ENU errors of baseline estimations [MADOCA]

Table 4 : RMS-ERRORS of baseline estimations

\begin{tabular}{|l|c|c|c|c|}
\hline \multicolumn{2}{|c|}{} & & \multicolumn{2}{c|}{ RMS [m] } \\
\hline \multirow{4}{*}{$u_{21}$} & \multirow{3}{*}{ Broadcast Ephemeris } & $\mathrm{E}$ & 0.0590 & \\
\cline { 3 - 4 } & & $\mathrm{N}$ & 0.0651 & 0.0938 \\
\cline { 3 - 4 } & \multirow{3}{*}{ MADOCA Products } & $\mathrm{U}$ & 0.1368 & \\
\cline { 3 - 4 } & & $\mathrm{E}$ & 0.0590 & \\
\cline { 3 - 4 } & & $\mathrm{N}$ & 0.0651 & 0.0938 \\
\cline { 3 - 4 } & & $\mathrm{U}$ & 0.1368 & \\
\hline
\end{tabular}

Baseline estimations for using both ephemeridesis are al- most same results such that the Eulers' angles in GNSS Gyro are same for applying both ephemeridesis.

\section{CONCLUSIONS}

We have presented VPPP algorithms with multiple antennas by applying MADOCA Products. When applying MADOCA Products, positioning accuracy is better than one applying the Broadcast Ephemeris. Namely, the accuracy 
of the positioning using the MADOCA Products is aproximately $0.5[\mathrm{~m}]$, better than one with comparing to using the Broadcast Ephemeris. However, baseline errors between two antennas for using both ephemeridesis are almost same. Therefore estimation results of Eulers' angles in GNSS Gyro are same for applying both ephemeridesis.

\section{REFERENCES}

[1] Y. Karatsu, M. Ozaki, Y. Kubo and S. Sugimoto: Further Studies on the VPPP Algorithms by using Multiple Antennas, Proc. of the 46th ISCIE Int. Symp. on Stochastic Systems Theory and Its Applications, pp. 171-179, Kyoto, Japan, Nov. (2014).

[2] Y. Karatsu, A. Mouri, Y. Kubo and S. Sugimoto: Further Development of VPPP Algorithms with Multiple Antennas, Proc. of the 28th Int. Tech. Meeting of The Satellite Division of the Institute of Navigation (ION GNSS+ 2015), pp. 1181-1192, Tampa, Florida, September (2015).

[3] G. Okuda, A. Mouri, H. Hasegawa, Y. Kubo and S. Sugimoto: VPPP Algorithms with Multiple Antennas and Highly Accurate Attitude Estimation by Ambiguity Resolution Methods, Proc. of the 30th Institute of Navigation ION GNSS+ (ION GNSS+'17), Portland Oregon (Sep. 2017).

[4] G. Okuda, H. Hasegawa, D. Mimura, Y. Kubo and S. Sugimoto: Advancement of Precise Positioning and GNSS Gyro Using Multiple GNSS Antennas (in Japanese), Proc. of the 62 Annual Conf. of ISCIE, Kyoto (May. 2018).

[5] S. Sugimoto: GNSS Positioning Algorithms based on GR Models, Section 7.3.1 of GPS Handbook (in Japanese) ed. by S. Sugimoto and R. Shibasaki, Asakura-Shoten, Tokyo, Japan (2010).

[6] Satoshi Kogure: On the developments of MADOCA and MADOCA-PPP, 2016, http://www.ic-madoca. org/documents/2_handout_JAXA.pdf

[7] GPAS: Quasi-Zenith Satellite System, Correction Data on Centimeter Level Augumentation Service for Experiment Data Format Specification, 2017, http://file.gpas. co.jp/L6E_MADOCA_DataFormat_E.pdf

[8] JAXA: Interface Specification for MADOCA-SEAD, 2017, https://ssl.tksc.jaxa.jp/madoca/public/ doc/Interface_Specification_A_en.pdf

[9] P. J. G. Teunissen: The Least-square Ambiguity Decorrelation Adjustment: A Method for Fast GPS Integer Ambiguity Estimation, J.Geodesy, Vol. 70, pp. 65-82, (2005).

[10] S. Fukuda: GNSS Applications to Attitude Determination Systems, Section 13 in GPS Handbook (in Japanese), pp. 306-330, ed. by S. Sugimoto and R. Shibasaki, AsakuraShoten, Tokyo, Japan (2010).

[11] R. G. Brown and P. Y. C. Hwang: Introduction to Random Signals and Applied Kalman Filtering, 3rd Edition, John Wiley \& Sons, New York (1997).

[12] A. Chabata, Y. Suzuki, Y. Kubo and S. Sugimoto: RTK-PPP Algorithms using GNSS Observables from Few Satellites, Proc. ION-GNSS 2012, Nashville, Tennessee, Sept. (2012).
[13] B. W. Parkinson and J. J. Spilker: The Global Positioning System: Theory and Applications, Vol. I, AIAA Publications (1996).

\section{Appendix}

Let us define the rotation angle $\phi$ and the normal vector $\mathbf{n}$ between two normalized baseline vectors $\mathbf{s}, \mathbf{t}$ :

$$
\mathbf{s}=\left(x_{0}, y_{0}, z_{0}\right), \quad \mathbf{t}=\left(x_{1}, y_{1}, z_{1}\right) .
$$

According to the Euler's principal rotation theorem, the position of $\mathbf{s}$ is moved to the position of $\mathbf{t}$ by the rotation of the angle $\phi$ around the normal vector which is called Euler axis. $\cos \phi$ is obtained from the inner product of two vectors $\mathbf{s}, \mathbf{t}$, and the normal vector $\mathbf{n}$ is obtained from the outer product of two vectors $\mathbf{s , t}$.

$$
\begin{aligned}
\cos \phi & =\mathbf{s} \cdot \mathbf{t} \\
& =x_{0} x_{1}+y_{0} y_{1}+z_{0} z_{1} \\
\mathbf{n} & =\left(n_{x}, n_{y}, n_{z}\right)^{\mathrm{T}}=\frac{\mathbf{s} \times \mathbf{t}}{\|\mathbf{s} \times \mathbf{t}\|} \\
& =\frac{1}{\sin \phi}\left(y_{0} z_{1}-z_{0} y_{1}, z_{0} x_{1}-x_{0} z_{1}, x_{0} y_{1}-y_{0} x_{1}\right) .
\end{aligned}
$$

From Eq.(95)-(96), we have following equations:

$$
\begin{aligned}
\cos \phi & =x_{0} x_{1}+y_{0} y_{1}+z_{0} z_{1} \\
n_{x} \sin \phi & =y_{0} z_{1}-z_{0} y_{1} \\
n_{y} \sin \phi & =z_{0} x_{1}-x_{0} z_{1} \\
n_{z} \sin \phi & =x_{0} y_{1}-y_{0} x_{1} .
\end{aligned}
$$

Transforming Eq.(97)-(100), $\left(x_{1}, y_{1}, z_{1}\right)$ are expressed by $\left(x_{0}, y_{0}, z_{0}\right),\left(n_{x}, n_{y}, n_{z}\right)$, and $\phi:$

$$
\begin{aligned}
& x_{1}=x_{0} \cos \phi+z_{0} n_{y} \sin \phi-y_{0} n_{z} \sin \phi, \\
& y_{1}=y_{0} \cos \phi-z_{0} n_{x} \sin \phi+x_{0} n_{z} \sin \phi, \\
& z_{1}=z_{0} \cos \phi+y_{0} n_{x} \sin \phi-x_{0} n_{y} \sin \phi,
\end{aligned}
$$

Eq.(101)-(103) are expressed by matrix form as follows:

$$
\left[\begin{array}{l}
x_{1} \\
y_{1} \\
z_{1}
\end{array}\right]=\left[\begin{array}{cccc}
x_{0} & 0 & z_{0} & -y_{0} \\
y_{0}-z_{0} & 0 & x_{0} \\
z_{0} & y_{0} & -x_{0} & 0
\end{array}\right]\left[\begin{array}{c}
\cos \phi \\
n_{x} \sin \phi \\
n_{y} \sin \phi \\
n_{z} \sin \phi
\end{array}\right]
$$

When we obtain two baseline vectors $\mathbf{s}, \mathbf{t}$, we estimate the parameters $\left(n_{x}, n_{y}, n_{z}\right), \cos \phi$, and $\sin \phi$. Then we derive the rotation matrix in Eq.(86) by the estimates. 